\title{
«Die Copy-paste-Mentalität ist gefährlich»
}

\section{Daniel Lüthi}

Freier Journalist und Fotograf, Medientrainer, Bern

Es beginnt mit einem Schokoladeherzlein, das der Chefarzt zum Kaffee in der Kantine reicht. Eine kleine, feine, offenbar typische Geste. Weiter geht es in seinem Büro, denn Michele Genoni will nicht im Operationssaal fotografiert werden. Das vermittle ein allzu technisches Bild seiner Tätigkeit. «Herzchirurgie ist nur zum Teil Handwerk», erklärt er. Womit wir bereits beim Herz seines beruflichen Credos sind.

\section{Spitzenmedizin und Spitzensport}

Immer gehe es darum, einen Menschen für eine Operation zu gewinnen, sagt Genoni. «Ich sehe mich als
Coach, als Trainer. Tore schiessen, gewinnen wollen ist Sache des Patienten.» Womit wir schon bei einem zweiten wichtigen Thema im Leben dieses Herzchirurgen sind: Eishockey. Alle drei Söhne waren Profis, jetzt steht noch der mittlere auf dem Eis, als Torhüter beim HC Davos. Der Spitzensport hat auch Vater Genoni geprägt, die Vergleiche kommen immer wieder. Auch eine Operation sei ein Mannschaftssport, sagt er, und: «Spitzensportler und Spitzenmediziner wandeln beide auf dem schmalen Grat zwischen Sieg und Niederlage, zwischen Erfolg und Misserfolg. Entscheidend ist die «mentalità vincente». Dass er für ‘Siegermentalität` den italienischen Ausdruck

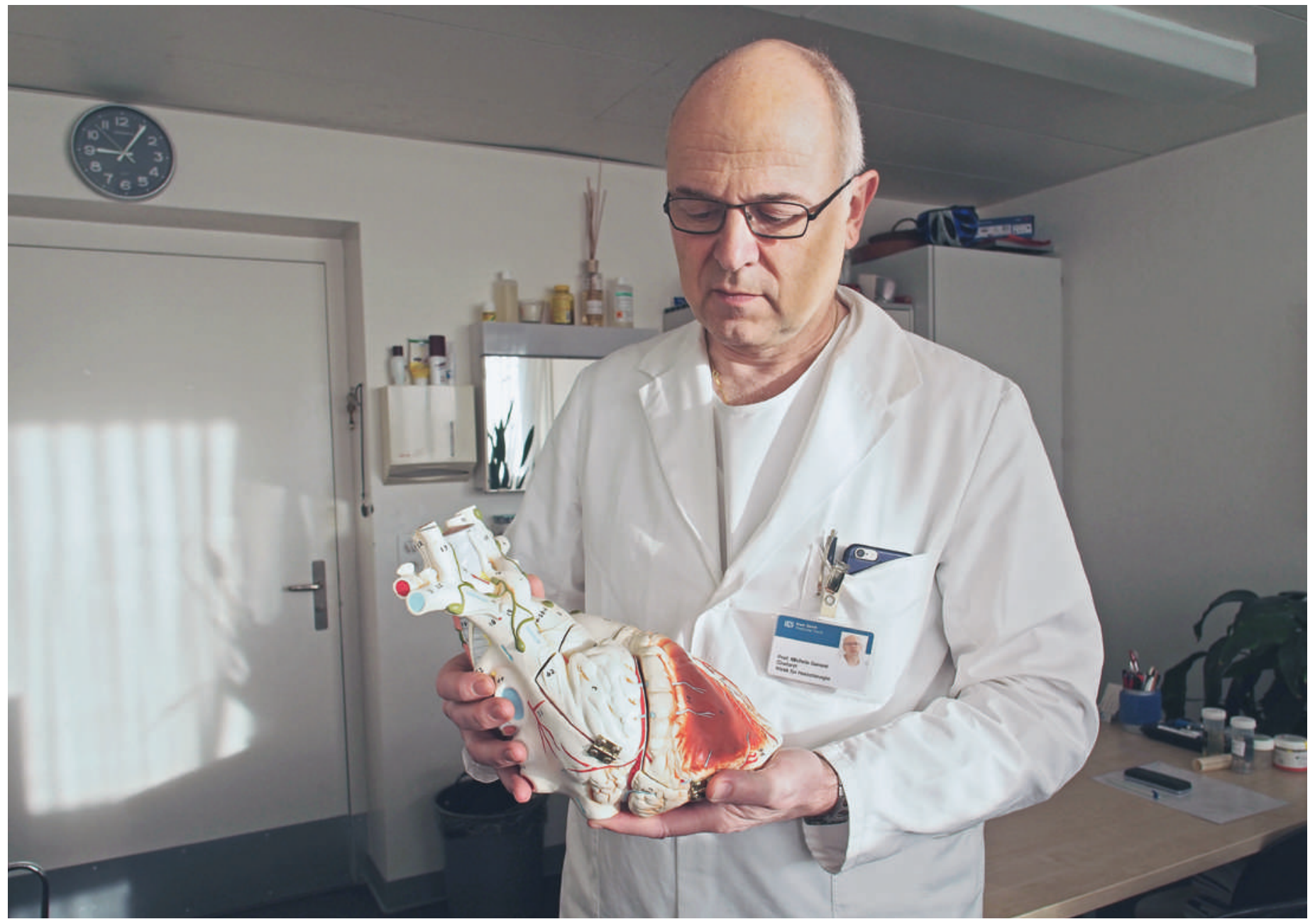


braucht, ist ebenfalls typisch. Beide Eltern von Michele Genoni sind Tessiner, sein Heimatort ist Semione im Bleniotal, ein Teil seines Herzens ist dort geblieben. "Ich wurde geprägt von der mediterranen Kultur.» Mehr auf die Leute zugehen, bedeute das, und eine gute Mischung zwischen Strukturiertheit und der Möglichkeit zur Spontaneität.

\section{Operieren am schlagenden Herz}

Zusammen mit drei anderen gut ausgebildeten Operateuren führt Genoni in seiner Klinik pro Jahr insgesamt rund 560 Herzoperationen durch, persönlich ist er an etwa 180 davon beteiligt. Spezialität des Teams sind Bypass-Operationen am schlagenden Herzen. «Das ist etwas anspruchsvoller als die Variante mit Herz-Lungen-Maschine, aber trotzdem weniger stressig, weil bei uns nicht so aufdringlich eine Uhr tickt und es nicht darum geht, möglichst schnell fertig zu sein.» Den Patienten komme diese Methode entgegen, weil sie für ihr Herz schonender sei und auch die Zeit in der Intensivstation verkürze.

\section{"Von Patienten werden wir oft hochgejubelt.»}

Eine solche Operation dauere zwischen dreieinhalb und fünf Stunden «und alle müssen genau wissen, wie's läuft». Und: Wichtig sei das stetige Training, wie im Sport. Oder wie beim Autofahren: «Wenn ich auf dem Weg ins Tessin immer die gleiche spezielle Strecke benütze und sie deshalb gut kenne, dann kann ich auch bei schlechtem Wetter dort durchfahren.» Praktisch alle minimalinvasiven Eingriffe, wie zum Beispiel Bypass-Operationen, werden im Triemlispital deshalb am schlagenden Herzen durchgeführt. Das muss einem Operateur doch das erhabene Gefühl vermitteln, im Zentrum tätig sein, an einem ganz entscheidenden Punkt des Lebens - ist das so? Ist die Herzchirurgie die Königsdisziplin der Medizin? Genoni sieht das ganz pragmatisch: "Wir sind in einem sehr technischen und sehr standardisierten Gebiet tätig. Wenn ich am Herz arbeite, sehe ich das Organ und die jeweilige Problematik. Aber klar: Von Patienten werden wir oft hochgejubelt, weil sie vor der Operation in der Regel grosse Angst haben und nach der Operation entsprechend erleichtert sind.»

\section{Der schmale Grat}

Aber da ist ja dieser schmale Grat zwischen Erfolg und Misserfolg, den er auch schon erwähnt hat, die Grenze zwischen Leben und Tod letztlich, die Möglichkeit einer Niederlage.

\section{Michele Genoni}

Prof. Dr. med. Michele Genoni wurde 1957 in Luzern geboren. Seine Jugend verbrachte er in Kilchberg $(\mathrm{ZH})$ und Sarnen (OW). Er studierte Medizin in Zürich und erwarb 1983 das eidgenössische Arztdiplom. 1984 bis 1991 bildete er sich weiter als Kardiologe und Chirurg in Bellinzona, Zürich und Lugano. 1992 kam er als Assistent von

Professor Marko Turina an die Klinik für Herzchirurgie des Universitätsspitals Zürich. 1994 bis 1999 war er hier Oberarzt, 1999 bis 2004 war er unter Turina Leitender Arzt für Herzchirurgie am Triemli-Spital Zürich, 2004 bis 2008 Klinikdirektor am Universitätsspital Zürich. Seit 2004 ist er Chefarzt der Klinik für Herzchirurgie am Stadtspital Triemli. Michele Genoni ist verheiratet und Vater von drei erwachsenen Söhnen. Er lebt in Kilchberg (ZH).

Oft sind es gerade in einer hochspezialisierten Umgebung ganz einfache Dinge, die entscheiden.

Ein negatives Paradebeispiel erlebte die Schweiz diesbezüglich im April 2004, als bei einer Herztransplantation im Universitätsspital Zürich die Blutgruppe verwechselt wurde. Die Tatsache, dass ausgerechnet diese Operation vom Schweizer Fernsehen ausführlich dokumentiert wurde, machte das Ganze nur noch schlimmer. Michele Genoni arbeitete damals zwar im Triemlispital, war aber Teil des Teams von Professor Turina. Entsprechend nahe geht ihm der Fall Voser heute noch. "In unserem Berufsalltag sehen wir immer wieder, dass sich Gewohnheiten einschleichen, die von anderen gedankenlos übernommen werden", kommentiert er. «Die Copy-paste-Mentalität ist gefährlich. Es ist gefährlich, dem anderen einfach zu glauben und es nicht zu wagen, Fragen zu stellen. Wir müssen ‘Tüpflischiisser» sein und immer alles selber nachkontrollieren.» Dem aber stehe etwas anderes im Weg: «Wir Herzchirurgen haben narzistische Grundzüge, und das kann zum Problem werden. Eine anerzogene Selbstsicherheit und die Tatsache, immer wieder hochgejubelt zu werden, können dazu führen, dass die Fehler- und die Kritikkultur leiden.» Er selber versuche, seine Mitarbeitenden zu einer kritischen Haltung sich selber, aber auch Vorgesetzten gegenüber, zu ermuntern. Einmal pro Monat veranstalte er in seiner Klinik ein CIRS-Meeting, wo alle aus kritischen Zwischenfällen lernen könnten - «70 Prozent der Meldungen kommen allerdings von mir.» Was zeigt, dass es sich vor allem der Chef leistet, Fehler zuzugeben. "Oh ja», sagt Genoni, «ich habe schon viele Fehler gemacht. Und ich habe auch schon Patienten verloren. Für meinen Seelenfrieden ist es wichtig, dass ich den Ursprung eines Fehlers finde und daraus lernen kann. Denn eine Niederlage schmerzt mich. Und 
das Schicksal, das deswegen ein Patient erleidet, bedrückt mich.»

Der Begriff «Niederlage» führt uns wieder in die Welt des Spitzensports. «Das habe ich von meinen Söhnen gelernt: Du musst immer besser werden wollen. Deshalb braucht es nach jedem Spiel - oder eben jeder Operation - eine gute Analyse, eine Besprechung in der Mannschaft.»

\section{Machtkämpfe statt Teamgeist}

Die Herzchirurgen in der Schweiz sind keine Mannschaft, da herrscht viel Konkurrenzdenken - Michele Genoni bestätigt dies: «Allein schon in Zürich gibt es diesen Machtkampf zwischen zwei öffentlichen Spitälern und drei herzchirurgischen Abteilungen einer Privatklinik, deshalb wäre es wichtig, dass sich die öffentlichen Spitäler zusammenschliessen würden. Denn die öffentliche Medizin arbeitet gegen eine Zweiklassenmedizin. Aber mit der Idee eines gemeinsamen Herzzentrums sind wir Anfang 2014 leider gescheitert. Es muss aber weiterhin eine Zusammenarbeit angestrebt werden.»

"Mit der Idee eines gemeinsamen Herzzentrums sind wir Anfang 2014 leider gescheitert.»

Auch auf nationaler Ebene seien Machtkämpfe leider allzu dominierend, sagt Genoni, der zurzeit als Präsident der Fachgesellschaft für Herzchirurgie amtet. «Es haben sich zu viele Partikularinteressen entwickelt, und es gibt zu viele Ressentiments. Wir Herzchirurgen müssen wieder eine gemeinsame Basis finden.» Dazu gehöre auch der Willen zur Konzentration: "18 Herzchirurgie-Zentren sind für dieses Land langfristig zu viel. Das Hauptproblem dabei sind jedoch nicht die Finanzen, es ist der Mangel an Fachpersonal, der sich abzeichnet.»

\section{Spitzenmedizin und Grundversorgung}

A propos Geld: Der Spitzenmedizin wird ja - insbesondere von den Grundversorgern - immer wieder vorgeworfen, im Verhältnis zu viele Ressourcen zu verschlingen. Zweierlei sagt Genoni dazu: «Ja, wir sind Teil der Spitzenmedizin. Die Infrastruktur, die wir brauchen, ist enorm, und das kostet viel.» Aber auch:
«Herzchirurgie ist Teil einer erweiterten Grundversorgung und gehört heute zum Standard. Natürlich kommen wir immer näher an das ethische Dilemma, was sinnvoll, beziehungsweise was machbar ist.» Vieles sehe anders aus, wenn es einen selber betreffe, fügt er schmunzelnd hinzu. «Im Übrigen geht der gesellschaftliche Zwang zur Ökonomisierung weitgehend an mir vorbei. Mein Auftrag ist es, die Lebensqualität und die Lebensprognose meiner $\mathrm{Pa}$ tientinnen und Patienten zu verbessern. Und dies haben alle zugute.» Also nicht nur Privatversicherte. «Ich selber operiere mehr Allgemeinversicherte als andere.»

Auch ist er ab und zu gerne mit dem «allgemeinen Volk» in einem ganz anderen Kontext zusammen, im Eishockeystadion zum Beispiel. «Leider kann ich aus Zeitgründen nicht mehr so viele Spiele besuchen wie früher. Aber wenn immer möglich, bin ich dabei. Und als unser Sohn mit dem HCD vor Weihnachten in Ambri spielte, war die halbe Genoni-Dynastie dort.»

\section{Ja, die Zeit}

Eigentlich hätte er mit zunehmendem Alter gerne mehr Zeit für sich selber gehabt. «Mit 60 wollte ich für mich die Viertagewoche einführen. Zurzeit sieht es allerdings eher nach mehr aus. Es ist nicht leicht, Musse zu finden.» Gleichzeitig aber ist es offenbar auch nicht leicht, sich eine Zeit mit mehr Zeit vorzustellen. «Meiner Pensionierung blicke ich mit Bedenken entgegen. Ich stelle mir die Frage, was ich ohne meine heutigen Strukturen machen werde.» Der Herzchirurg Michele Genoni wird sich ein Herz fassen müssen.

Vorderhand macht er es symbolisch: In seinem Büro steht nämlich, verankert in einem Sockel, ein Modellherz. Genoni nimmt es, fast wie ein Baby, in die Hand. Ein Spruch kommt ihm dabei in den Sinn: «Fass dir ein Herz, sagte der Herzlose, und griff ins Leere.»

\footnotetext{
Die nächste "Begegnung mit ...»

Am Ende jeden Monats stellt die Schweizerische Ärztezeitung eine Persönlichkeit vor, die sich im Gesundheitswesen engagiert. Im März schildert Daniel Lüthi seine Begegnung mit Prof. Dr. med. Jean-François Dufour, Chefarzt Hepatologie am Berner Inselspital.
} 